\title{
PROTOCOL
}

\section{Interventions Targeting the Prescribing and Monitoring of Vancomycin for Hospitalized Patients: A Systematic Review Protocol}

\author{
Cameron J. Phillips (D) - Alice J. Wisdom - Ross A. McKinnon • \\ Richard J. Woodman · David L. Gordon
}

Received: August 30, 2017 / Published online: October 27, 2017

(C) The Author(s) 2017. This article is an open access publication

\section{ABSTRACT}

Introduction: Vancomycin remains one of our essential antibiotics after fifty years of treating serious infections such as methicillin-resistant Staphylococcus aureus. Vancomycin, unlike many other antibiotic agents, requires individualized dosing and monitoring of serum drug levels to ensure it is efficacious, to minimize toxicity, and to limit the development of

Enhanced content To view enhanced content for this article go to http://www.medengine.com/Redeem/ 38CCF060756CE31D.

Electronic supplementary material The online version of this article (doi:10.1007/s40121-017-0177-7) contains supplementary material, which is available to authorized users.

C. J. Phillips $(\bowtie)$

SA Pharmacy, Flinders Medical Centre, Adelaide, Australia

e-mail: cameron.phillips@sa.gov.au

C. J. Phillips · R. A. McKinnon · R. J. Woodman ·

D. L. Gordon

College of Medicine and Public Health, Flinders

University, Adelaide, Australia

C. J. Phillips · R. A. McKinnon

School of Pharmacy and Medical Sciences,

University of South Australia, Adelaide, Australia

A. J. Wisdom

SA Pharmacy, Lyell McEwin Hospital, Adelaide, Australia antibiotic resistance. These issues have led to numerous vancomycin clinical practice guidelines being published in recent years including several key national guidelines. Significant resources are invested during the development of such guidelines; however, there is often little or no information about how such guidelines or other vancomycin practice improvement initiatives should be implemented. The aim of this systematic review is to identify and evaluate the effect of interventions using education, guideline implementation, and dissemination of educational resources that have sought to improve therapeutic drug monitoring and dosing of vancomycin.

Methods: A systematic review of the literature will be conducted for RCTs and observational studies where a vancomycin guideline or

\section{R. A. McKinnon}

Flinders Centre for Innovation in Cancer, Flinders University, Adelaide, Australia

\section{R. J. Woodman}

Flinders Centre for Epidemiology and Biostatistics, Flinders University, Adelaide, Australia

D. L. Gordon

SA Pathology, Microbiology and Infectious Diseases, Flinders Medical Centre, Adelaide, Australia

D. L. Gordon Division of Medicine, Flinders Medical Centre, Adelaide, Australia 
practice improvement initiative has been implemented. Electronic databases to be searched are PubMed, Medline, CINAHL, EMBASE and the Cochrane Library of Systematic Reviews. The population will be patients who have had intravenous vancomycin prescribed and monitored in hospital. The interventions will be education, implementation of guidelines or protocols, dissemination of educational materials (printed or electronic) or multifaceted interventions of the above. The comparator will be patients who have had standard-care prescribing and monitoring of vancomycin. Outcomes will be changes in prescribing and ordering of vancomycin serum tests, and serum levels attained in patients as well as reported nephrotoxicity. Two reviewers will be involved in the quality assessment and extraction of data. The Scottish Intercollegiate Guidelines Network checklist for RCTs will be used. Studies that are not randomized will be assessed for quality using the validated ROBINS-I (risk of bias in non-randomized studies of interventions) tool.

Discussion: This systematic review will identify interventions that have been used to implement guidelines and clinical practice initiatives for vancomycin. The findings of this review may be informative to those involved with the implementation of vancomycin clinical practice guidelines.

Systematic review registration: PROSPERO: CRD42016049147.

Keywords: Education; Guideline; Implementation; Intervention; Protocol; Vancomycin

\section{INTRODUCTION}

While vancomycin has been used for nearly 60 years, it remains the principal treatment for infection caused by serious Gram-positive bacteria such as methicillin-resistant Staphylococcus aureus (MRSA) [1]. Vancomycin, unlike many other antibiotics, has a number of special considerations, such as the requirement for individualization of dosing and serum drug monitoring to ensure efficacy, minimize toxicity and limit the development of bacterial resistance [2-4].
These factors, in addition to increasing concerns about antimicrobial resistance $[5,6]$ and the need to prolong the life of our existing antibiotics, have led to the publication of a number of vancomycin guidelines [7], including important national guidelines for the dosing and or monitoring of vancomycin from the United States (US), Japan and China [8-10]. Significant effort and resources are invested in the process and preparation of such high-quality national guidelines, which are endorsed by peak professional societies in their respective countries $[11,12]$. These documents provide much needed contemporary guidance on the appropriate use of vancomycin; however, there is a paucity of information about how these vancomycin guidelines and their contents should be best disseminated and implemented into practice to achieve the intended outcomes for clinicians and patients. Only one guideline, by the Chinese Pharmacological Society [10], includes some information about implementation. The implementation details associated with this guideline propose promotion via conferences, education sessions for physicians, pharmacists and nurses, and research to evaluate both the implementation and impact of the guideline on vancomycin therapeutic drug monitoring (TDM) [13].

There are numerous reports in the medical literature that highlight clinicians lack of knowledge of the contents of key guidelines in addition to an often low uptake of guidelines [14-16]. To combat this issue, adoption strategies have been recommended by a number of prominent organizational developers of guidelines such as the Australian National Health and Medical Research Council (NHMRC) $[17,18]$, the United Kingdom's National Institute for Health and Clinical Excellence (NICE) [19], the Scottish Intercollegiate Guideline Network (SIGN) [20], and the US Institute of Medicine (IOM) [21]. While it is prudent that any plan to implement a guideline or practice change should include an assessment of the barriers and enablers [22], there are common implementation strategies recommended by these organizations, which are widely employed. Such strategies include the provision of education about the guideline and its recommendations [23]. Educational meetings 
have demonstrated changes in practice measures between $1.8 \%$ and $15.9 \%$ [24], while dissemination of guidelines and educational supporting material have been shown to have a median $8.1 \%$ improvement on care [25], although there have been recent concerns about the effectiveness of the latter [26]. Determination of the relative effectiveness of these strategies to promote the implementation of guidelines or practice change initiatives for vancomycin is important to prudently allocate supportive resources. While a systematic review on guidelines for TDM of vancomycin has been published [7], the current review aims to identify and evaluate the effect of interventions employing education, guideline implementation and dissemination of educational resources on the therapeutic drug monitoring and dosing of vancomycin.

\section{METHODS}

The steps of the systematic review to be conducted will be defining the inclusion criteria and exclusion criteria, searching for and capturing studies, and identifying studies that address the review question and are in accordance with the criteria. Defined data will be extracted and compiled. This systematic review protocol will follow the Preferred Reporting Items for Systematic Review and Meta-Analysis Protocols (PRISMA-P) 2015 statement [27, 28]. The PRISMA-P 2015 checklist for this review accompanies this protocol as Supplementary material 1.

\section{Research Question}

This review aims to systematically identify and determine the effect of interventions that have targeted the therapeutic drug monitoring and dosing of the intravenous antibiotic vancomycin.. The specific review question is:

Do interventions (alone or in combination) involving; education, implementation of guidelines or protocols, or dissemination of educational materials (printed or electronic) improve the prescribing, monitoring and safety of vancomycin?

\section{Population, Interventions, Comparator and Outcome (PICO)}

The review populations, interventions, comparator group and outcomes [29], to be assessed in the systematic review are presented in Table 1.

\section{Selection of Studies and Inclusion/ Exclusion Criteria}

A preliminary search suggests that there are limited RCTs on this topic, so observational, including before-after studies and interrupted time series studies, will also be included in addition to RCTs. The review will include studies that have employed documented implementation strategies for vancomycin guidelines and protocols, educational interventions (face-to-face or electronic, disseminations of educational materials (printed or electronic) or multifaceted strategies using a combination of these. Studies to be excluded will be those using population pharmacokinetic modeling of guidelines or protocols, those comparing one explicit guideline directly against another (e.g., continuous versus intermittent dosing), those with no comparison to control or baseline data, and those where the post-implementation assessment excluded patients who were not dosed in accordance with the new guideline (as this may bias and misrepresent uptake of the guideline). Studies will also be excluded if they focus solely on indication for vancomycin or duration of usage. Studies involving oral vancomycin for Clostridium difficile infection will be excluded as this therapy does not involve TDM.

\section{Search Strategy and Data Storage}

The search strategy was developed in collaboration with an academic medical librarian experienced in conducting searches for systematic reviews. Search strategies will employ medical subject headings (MeSH) [30], and key words pertaining to the research question. The electronic database search was initially developed for Ovid MEDLINE (full search strategy presented as Supplementary material 2). The 
Table 1 PICO framework

\begin{tabular}{ll}
\hline $\begin{array}{l}\text { Population } \\
\text { Interventions }\end{array}$ & $\begin{array}{l}\text { Patients who have had vancomycin prescribed and monitored in hospital } \\
\text { electronic) or multifaceted interventions of the above }\end{array}$ \\
Comparators & $\begin{array}{l}\text { Standard care prescribing and monitoring of vancomycin } \\
\text { Outcomes }\end{array}$ \\
Prescribing The proportion of patients prescribed loading doses, and prescribed maintenance doses \\
appropriate for renal function \\
Monitoring The proportion of vancomycin blood levels drawn at appropriate times, attaining specified target \\
ranges, and in levels outside specified ranges \\
Safety Frequency of reported nephrotoxicity (increase in serum creatinine of $0.5 \mathrm{mg} / \mathrm{dL}$ or $>50 \%$ from \\
baseline on $\geq 2$ or more consecutive measurements) after 2 or more days of vancomycin [8]
\end{tabular}

search strategy was then adapted for PubMed, EMBASE (Excerpta Medica Database), CINAHL (Cumulative Index to Nursing and Allied Health Literature) and the Cochrane Library of Systematic Reviews. The search will be filtered to capture articles in the English language only. As vancomycin was first licensed with the US Food and Drug Administration in the 1950s, the search strategy will span all articles in the respective databases from inception. To further the search strategy, any relevant studies identified by members of the review team will be captured. The search will be re-performed prior to closing the review to ensure any recently published articles are captured. Publications will be stored in in a dedicated electronic library using EndNote X7.7 referencing software (Thompson Reuters, 2016), with duplicate references to be removed. Data collection will be performed using Microsoft Excel (Microsoft, 2017).

\section{Data Analysis and Synthesis}

The preliminary screening of captured articles will be performed to determine if the titles or abstracts address the review question. A second reviewer will independently review articles to determine if they are in agreement with the suitability of selected articles. Any differences will be resolved through discussion with a third member of the review team. The following stage will be accessing full text articles to determine eligibility for final inclusion, when a second reviewer will independently check that they agree with the identified articles. Any disagreement will be resolved by a third member of the review team. An assessment of the quality of articles will be performed. The SIGN checklist for RCTs will be used [20]. Studies that are not randomized will be assessed using the validated tool ROBINS-I (Risk of bias in non-randomized studies of interventions) [31].

Data variables to be collected are study demographics, authors, year, country, care setting (unit or ward) in hospital, type of study, intervention type and description of intervention, intended effect of intervention, use of any theory for the intervention, learning objectives, materials used, educational strategies, schedule, instructions and modes used, use of incentives and environment [32]. Data for outcomes will be authors' results for vancomycin prescribing, drug monitoring and nephrotoxicity. This article does not contain any new studies with human or animal subjects performed by any of the authors.

\section{DISCUSSION}

Studies have demonstrated hospital doctors do not prescribe antibiotics appropriately nearly half of the time [33], and one-quarter of hospitals in Australia have been reported as non-adherent to guidelines [34]. Determination of the 
strategies that promote effective implementation should be a fundamental component of guideline development and practice improvement initiatives. The published literature on vancomycin prescribing and monitoring shows that there is considerable room for improvement for this half-century-old antibiotic. The findings from this systematic review will be summarized in tabular format providing ready interpretation and comparison of studies. We will provide a narrative synthesis of the findings from included studies structured around the type of intervention, prescriber and population characteristics and outcomes. We will also discuss the strengths and limitations of included studies. We elected not to measure clinical efficacy or microbiological outcomes, as we wanted to focus on outcomes pertaining specifically to dosing, TDM and toxicity which are highly appropriate as these are directly related to interventions providing guidance, education or dissemination of resources seeking to improve vancomycin dosing and TDM and to limit toxicity. This review will be informative in providing guidance on how successful the examined interventions are in effecting appropriate prescribing and monitoring of vancomycin. The findings of this review will help those seeking to improve the clinical use of vancomycin by selecting effective interventions to implement guidelines or other practice improvement initiatives.

\section{ACKNOWLEDGEMENTS}

No funding or sponsorship was received for this study protocol or publication of this article. The article processing charges were funded by the authors. All named authors meet the International Committee of Medical Journal Editors (ICMJE) criteria for authorship for this manuscript, take responsibility for the integrity of the work as a whole, and have given final approval for the version to be published. The authors would like to thank Leila Mohammadi, Medical Liaison Librarian, Gus Fraenkel Medical Library, Flinders University, Adelaide, Australia, for her expertise and guidance assisting in the development of search strategies for multiple databases.
Disclosures. Cameron J. Phillips, Alice J. Wisdom, Ross A. McKinnon, Richard J. Woodman, and David L. Gordon have nothing to disclose relevant to this work.

Compliance with Ethics Guidelines. This protocol is based on preparing to search for previously conducted studies and does not involve any new human or animal subjects performed by the authors.

Open Access. This article is distributed under the terms of the Creative Commons Attribution-NonCommercial 4.0 International License (http://creativecommons.org/licenses/ by-nc/4.0/), which permits any noncommercial use, distribution, and reproduction in any medium, provided you give appropriate credit to the original author(s) and the source, provide a link to the Creative Commons license, and indicate if changes were made.

\section{REFERENCES}

1. Rybak MJ, Rotschafer JC, Rodvold KA. Vancomycin: over 50 years later and still a work in progress. Pharmacotherapy. 2013;33:1253-5.

2. Rybak M. The pharmacokinetic and pharmacodynamic properties of vancomycin. Clin Infect Dis. 2006;42:S35.

3. Giuliano C, Haase KK, Hall R. Use of vancomycin pharmacokinetic-pharmacodynamic properties in the treatment of MRSA infections. Expert Rev Anti Infect Ther. 2010;8:95-106.

4. Howden BP, Davies JK, Johnson PD, Stinear TP, Grayson ML. Reduced vancomycin susceptibility in Staphylococcus aureus, including vancomycin-intermediate and heterogeneous vancomycin-intermediate strains: resistance mechanisms, laboratory detection, and clinical implications. Clin Microbiol Rev. 2010;23:99-139.

5. Kelly R, Zoubiane G, Walsh D, Ward R, Goossens H. Public funding for research on antibacterial resistance in the JPIAMR countries, the European Commission, and related European Union agencies: a systematic observational analysis. Lancet Infect Dis. 2016;16:431-40.

6. World Health Organization. Antimicrobial resistance: global report on surveillance. World Health 
Organization; 2014. http://apps.who.int/iris/ bitstream/10665/112642/1/9789241564748_eng.pdf. Accessed Aug 13, 2017.

7. Ye ZK, Li C, Zhai SD. Guidelines for therapeutic drug monitoring of vancomycin: a systematic review. PLoS ONE. 2014;9(6):e99044.

8. Rybak MJ, Lomaestro BM, Rotschafer JC, Moellering RC, Craig WA, Billeter M, et al. Vancomycin therapeutic guidelines: a summary of consensus recommendations from the Infectious Diseases Society of America, the American Society of Health-System Pharmacists, and the Society of Infectious Diseases Pharmacists. Clin Infect Dis. 2009;49(3):325-7.

9. Matsumoto K, Takesue Y, Ohmagari N, Mochizuki T, Mikamo H, Seki M, et al. Practice guidelines for therapeutic drug monitoring of vancomycin: a consensus review of the Japanese Society of Chemotherapy and the Japanese Society of Therapeutic Drug Monitoring. J Infect Chemother. 2013;19:365-80.

10. Ye ZK, Chen YL, Chen K, Zhang XL, Du GH, He B, et al. Therapeutic drug monitoring of vancomycin: a guideline of the Division of Therapeutic Drug Monitoring, Chinese Pharmacological Society. J Antimicrob Chemother. 2016;71:3020-5.

11. Qaseem A, Forland F, Macbeth F, Ollenschlager G, Phillips S, van der Wees P. Guidelines International Network: toward international standards for clinical practice guidelines. Ann Intern Med. 2012;156:525-31.

12. Steinbrook R. Improving clinical practice guidelines. JAMA Intern Med. 2014;174:181.

13. Ye ZK, Chen K, Chen YL, Zhai SD. A protocol for developing a clinical practice guideline for therapeutic drug monitoring of vancomycin. J Huazhong Univ Sci Technol Med Sci. 2016;36:469-72.

14. Cabana MD, Rand CS, Powe NR, Wu AW, Wilson $\mathrm{MH}$, Abboud PA, et al. Why don't physicians follow clinical practice guidelines? A framework for improvement. JAMA. 1999;282:1458-65.

15. Lomas J, Anderson GM, Domnick-Pierre K, Vayda E, Enkin MW, Hannah WJ. Do practice guidelines guide practice? The effect of a consensus statement on the practice of physicians. $\mathrm{N}$ Engl J Med. 1989;321:1306-11.

16. Mol P, Rutten W, Gans R, Degener JE, Haaijer-Ruskamp FM. Adherence barriers to antimicrobial treatment guidelines in teaching hospital, the Netherlands. Emerg Infect Dis. 2004;10:522-5.

17. National Health and Medical Research Council (NHMRC). A guide to the development, implementation and evaluation of clinical practice guidelines. Canberra: National Health and Medical Research Council; 1999.

18. National Health and Medical Research Council. Procedures and requirements for meeting the 2011 NHMRC standard for clinical practice guidelines. Melbourne: National Health and Medical Research Council; 2011.

19. National Institute for Health and Clinical Excellence (NICE). PMG6 the guidelines manual. London: National Institute for Health and Clinical Excellence; 2012. https://www.nice.org.uk/process/ pmg6/resources/the-guidelines-manual-pdf-2007970 804933. Accessed Aug 13, 2017.

20. Scottish Intercollegiate Guideline Network. SIGN 50: a guideline developers' handbook 2015. http:// www.sign.ac.uk/assets/sign50_2015.pdf. Accessed Aug 13, 2017.

21. Graham R, Mancher M, Wolman DM, Greenfield S, Steinberg E. Clinical practice guidelines we can trust. Washington D.C.: National Academies Press; 2011.

22. Grol R, Grimshaw J. From best evidence to best practice: effective implementation of change in patients' care. Lancet. 2003;362:1225-30.

23. O'Brien MA, Rogers S, Jamtvedt G, Oxman AD, Odgaard-Jensen J, Kristoffersen DT, et al. Educational outreach visits: effects on professional practice and health care outcomes. Cochrane Database Syst Rev. 2007;(4):CD000409. doi:10.1002/ 14651858.CD000409.pub2.

24. Forsetlund L, Bjorndal A, Rashidian A, Jamtvedt G, $\mathrm{O}^{\prime}$ Brien MA, Wolf F, et al. Continuing education meetings and workshops: effects on professional practice and health care outcomes. Cochrane Database Syst Rev. 2009;(2):Cd003030. doi:10. 1002/14651858.CD003030.pub2.

25. Grimshaw JM, Thomas RE, MacLennan G, Fraser C, Ramsay CR, Vale L, et al. Effectiveness and efficiency of guideline dissemination and implementation strategies. Health Technol Assess. 2004;8:iii-iv, 1-72.

26. Grudniewicz A, Kealy R, Rodseth RN, Hamid J, Rudoler D, Straus SE. What is the effectiveness of printed educational materials on primary care physician knowledge, behaviour, and patient outcomes: a systematic review and meta-analyses. Implement Sci. 2015;10:164.

27. Shamseer L, Moher D, Clarke M, Ghersi D, Liberati A, Petticrew M, et al. Preferred reporting items for systematic review and meta-analysis protocols (PRISMA-P) 2015: elaboration and explanation. BMJ. 2015;349:g7647. 
28. Moher D, Shamseer L, Clarke M, Ghersi D, Liberati A, Petticrew $M$, et al. Preferred reporting items for systematic review and meta-analysis protocols (PRISMA-P) 2015 statement. Syst Rev. 2015;4:1.

29. Schardt C, Adams MB, Owens T, Keitz S, Fontelo P. Utilization of the PICO framework to improve searching PubMed for clinical questions. BMC Med Inform Decis Mak. 2007;7:16.

30. Kim S, Yeganova L, Wilbur WJ. Meshable: searching PubMed abstracts by utilizing $\mathrm{MeSH}$ and MeSH-derived topical terms. Bioinformatics. 2016;32:3044-6.

31. Sterne JA, Hernan MA, Reeves BC, Savovic J, Berkman ND, Viswanathan M, et al. ROBINS-I: a tool for assessing risk of bias in non-randomised studies of interventions. BMJ. 2016;355:i4919.
32. Phillips AC, Lewis LK, McEvoy MP, Galipeau J, Glasziou P, Moher D, et al. Development and validation of the guideline for reporting evidence-based practice educational interventions and teaching (GREET). BMC Med Educ. 2016;16:237.

33. Davey P, Brown E, Charani E, Fenelon L, Gould IM, Holmes $\mathrm{A}$, et al. Interventions to improve antibiotic prescribing practices for hospital inpatients. Cochrane Database Syst Rev. 2017;2:CD003543. doi:10.1002/14651858.CD003543.pub4.

34. Australian Commission on Safety and Quality in Health Care (ACSQHC). AURA 2017: second Australian report on antimicrobial use and resistance in human health. Sydney: ACSQHC; 2017. http:// www.safetyandquality.gov.au/antimicrobial-useandresistance-in-australia/resources-page/. Accessed Aug 13, 2017. 